\title{
Maduración de Dientes Permanentes en Niños Venezolanos entre 3 y 6 años: Aplicabilidad en la Estimación de la Edad Dental con Fines Forenses
}

\author{
Permanent Teeth Maturation in 3-6 Year Old Venezuelan Children: \\ Applicability in Dental Age Estimation with Forensic Purposes
}

\author{
Alice Leonela Carrizo'; Maira Lisbet Quevedo-Piña ${ }^{2}$ \& Ana Isabel Ortega-Pertuz ${ }^{3}$
}

\begin{abstract}
CARRIZO, A. L.; QUEVEDO-PIÑA, M. L. \& ORTEGA-PERTUZ, A. I. Maduración de dientes permanentes en niños venezolanos entre 3 y 6 años: aplicabilidad en la estimación de la edad dental con fines forenses. Int. J. Odontostomat., 14(3):430-441, 2020.
\end{abstract}

RESUMEN: El objetivo de este trabajo fue estudiar la maduración de dientes permanentes en niños venezolanos entre tres y seis años de edad y su aplicabilidad en la estimación de la edad dental (ED) con fines forenses. Se realizó un estudio descriptivo, retrospectivo, transversal y de campo. La muestra consistió en 107 radiografías panorámicas de individuos venezolanos (56 mujeres y 51 hombres). Se asignaron los estadios de maduración a los dientes permanentes de los cuatro cuadrantes de acuerdo al método de Demirjian et al. (1973) original, mientras que la ED fue calculada por el método de Demirjian et al. y sus modificaciones (Demirjian \& Goldstein, 1976), Willems et al. (2001) y Chaillet et al. (2005). Las mujeres alcanzaron los estadios de maduración a edades más tempranas que los hombres. Se observó en el total de la muestra la sobre-estimación de la edad cronológica (EC) por todos los métodos, siendo el método de Chaillet et al. el más preciso. Al estudiar los sexos por separado, el método de Chaillet et al. presentó la menor diferencia EC-ED para los hombres y el de Chaillet et al. en las mujeres. El método de Demirjian et al., original mostró la mayor diferencia EC-ED para ambos sexos. Los métodos de Chaillet et al. y Willems et al., son aplicables con fines de determinación de la edad en la muestra estudiada, siendo su utilidad dependiente del sexo. dientes.

PALABRAS CLAVE: odontología forense, maduración dental, edad dental, estimación de la edad por los

\section{INTRODUCTION}

La ED representa un indicador de maduración de gran utilidad, pues puede ser aplicada clínicamente para establecer si el individuo se encuentra dentro de los parámetros normales para su grupo de edad, siendo esto un dato importante para la planificación de determinados tratamientos, así como un complemento diagnóstico en la detección de posibles alteraciones del crecimiento (Cruz-Landeira et al., 2010; NikHussein et al., 2011; Urzel \& Bruzek, 2013; Medina \& Blanco, 2014; Mohammed et al., 2014; Onat Altan et al., 2016). Otra utilidad de la ED está orientada hacia la Odontología Forense, en la cual constituye en oca- siones, la única forma de aproximación fiable para la determinación de la edad en individuos vivos o fallecidos que no cuenten con documentación válida para su identificación (Willems et al., 2001; Schmeling et al., 2007).

En general, se emplean dos enfoques para el estudio del desarrollo de la dentición permanente. El primero se centra en la valoración de la erupción dental cuando el diente es visible en la cavidad bucal, este evento puede ser influenciado por factores locales como anquilosis, extracción temprana o tardía de los dientes

\footnotetext{
${ }^{1}$ División de estudios para Graduados, Facultad de Odontología, Universidad del Zulia. Maracaibo, estado Zulia, Venezuela.

${ }^{2}$ Cátedra de Imagenología, Departamento de Ciencias Estomatoquirúrgicas, Facultad de Odontología, Universidad de Carabobo, Valencia, estado Carabobo, Venezuela.

${ }_{3}^{3}$ Área de Odontología Forense, Instituto de Investigaciones, Facultad de Odontología, Universidad del Zulia. Maracaibo, estado Zulia, Venezuela.
} 
temporales, así como la retención o apiñamiento de los dientes permanentes (Mohammed et al.). El segundo, es el estudio de la mineralización dental en radiografías, desde la formación de la corona hasta el cierre apical (Demirjian et al.; Demirjian \& Goldstein; Willems et al.; Cruz-Landeira et al.); éste ha demostrado ser menos variable y más controlado por la genética que por factores ambientales, .lo cual lo hace más confiable que la erupción dentaria en la predicción de la edad (Demirjian et al.; Demirjian \& Goldstein; Lee et al., 2008; Cruz-Landeira et al.; Nik-Hussein et al.; Willems et al.; Djukic et al., 2013; Galic' et al., 2013; Mohammed et al.; Aissaoui et al., 2016).

Varios métodos se han desarrollado con la finalidad de expresar el desarrollo dental en términos de edad. Entre ellos uno de los más utilizado es el propuesto por Demirjian et al. en 1973 (DEMO), el mismo se basa en la evaluación de los siete dientes inferiores izquierdos (excluyendo el tercer molar) a los cuales se les asignan estadios de desarrollo denominados de la $\mathrm{A}-\mathrm{H}$ que representan la mineralización de la corona hasta el cierre del ápice. Para la determinación de la ED se otorga una puntuación a cada estadio asignado a los dientes estudiados, posteriormente se suma las distintas puntuaciones para obtener un índice maduración dentaria (IMD) que es convertido en ED mediante el uso de tablas específicas para cada sexo.

Sin embargo, diversos estudios han indicado que al aplicar DEMO se ha observado una sistemática sobre-estimación de la edad, lo que ha sido atribuido a las distintas condiciones bio-psicosociales en las que se desarrolló la muestra franco-canadiense empleada para la construcción de las tablas de referencia del método y de los individuos en los cuales el mismo había sido utilizado (Chaillet et al., 2005; Lee et al.; Liversidge et al., 2010; Nur et al., 2012; Galic et al.; Urzel \& Bruzek; Ambarkova et al., 2014; Aissaoui et al.; Hegde et al., 2017). En 1976, Demirjian \& Goldstein realizaron una modificación al trabajo original de Demirjian et al., con el propósito de presentar nuevas tablas de referencia para el método que consideraba los siete dientes mandibulares izquierdos (DEM7AL), y diseñaron dos métodos adicionales donde se estudian cuatro dientes inferiores izquierdos, a saber: a. Técnica modificada (DEM4), donde se observa la maduración del primer y segundo molar y de ambos premolares inferiores izquierdos; $b$. Técnica alternativa (DEM4AL), en la cual se evalúan el segundo molar, los dos premolares y el incisivo central inferior izquierdos. Se presentan para todos los métodos propuestos, las tablas de puntuaciones así como los gráficos de percentiles para la maduración dental, los cuales se emplean para la estimación de la ED.

Por su parte, Willems et al., en 2001, propusieron tablas de referencias para la estimación de ED, basadas en el estudio de la maduración dental de 2116 niños belgas con edades entre los tres y 16 años de edad. Los autores emplean los mismos estadios de maduración de DEMO, pero las tablas de conversión de estadios a puntuaciones están expresadas en años, por lo que al sumarlas se calcula directamente la ED sin el paso intermedio de obtener un IMD como en el método original, lo cual simplifica su aplicación. En 2005, Chaillet et al. (CHA) construyeron tablas de referencias elaboradas con ocho muestras provenientes de distintos países (Australia, Bélgica, Canadá, Corea, Inglaterra, Finlandia, Francia, y Suecia), esperando mejorar la precisión en la estimación de la edad, cuando se aplicaran a individuos con origen étnico desconocido.

En particular, los sujetos entre los tres y seis años de edad experimentan durante este periodo, cambios importantes en el complejo maxilofacial, es una época clave para la detección de maloclusiones relacionadas con hábitos parafuncionales, así como lesiones en los órganos dentarios asociadas con caries y traumatismo, de allí la necesidad de conocer el patrón de desarrollo de la dentición permanente, lo cual es un factor a considerar en la instauración de la terapéutica adecuada (Águila \& Enlow, 1993; Gardner et al., 1995). Por otro lado, durante los primeros años de vida el individuo es particularmente vulnerable a situaciones de maltrato y/o tráfico humano, es estas circunstancias es probable que carezca de documentos válidos de identificación; de igual manera, en las regiones inmersas en conflictos bélicos o condiciones socio-económicas adversas, es común que se produzcan movimientos migratorios, por tal motivo, conocer la EC aproximada de pacientes con fecha de nacimiento desconocida, es una problemática a considerar desde el punto de vista legal (Schmeling et al.; Herdge et al.)

En Venezuela, se ha observado la sobre-estimación de la edad al aplicar el método de Demirjian et al. y Demirjian \& Goldstein, en muestras provenientes del estado Zulia (Martínez Gutiérrez \& Ortega-Pertuz, 2017; Ortega-Pertuz et al., 2018; Ortega-Pertuz \& Piña, 2020) y la zona metropolitana de Caracas (Medina \& Blanco) mientras que en individuos originarios del es- 
tado Mérida (Cruz-Landeira et al.), región andina del país, la edad fue subestimada. Con relación al método de Willems et al., se verificó una sub-estimación de la edad en los individuos provenientes de Caracas (Medina \& Blanco), en cuanto que en los zulianos los resultados variaron de acuerdo al sexo (Ortega-Pertuz \& Piña). Para Chaillet et al. se ha evidenciado una subestimación de la edad en andinos (Cruz-Landeia et al.) y hasta los 15 años de edad en una muestra de zulianos (Ortega-Pertuz et al.). Sin embargo, es importante señalar que la inclusión de individuos entre los tres y seis años edades en investigaciones sobre el desarrollo dental y su utilidad en la estimación de la edad, se ve limitada debido a la disponibilidad de las radiografías panorámicas, lo que se relaciona con la colaboración y tolerancia del paciente al procedimiento, así como su indicación con fines clínicos (Lee et al.; Galic et al.). Considerando lo anteriormente expuesto, el objetivo de la investigación fue estudiar la maduración de dientes permanentes en niños venezolanos entre los tres y seis años de edad, así como su aplicabilidad en la estimación de la ED con fines forenses, utilizando los métodos de Demirjian et al., Demirjian \& Goldstein, Willems et al. y Chaillet et al.

\section{MATERIAL Y METODO}

Se realizó un estudio descriptivo, transversal y de campo. La población correspondió al total de radiografías panorámicas de pacientes en edad preescolar que pertenecen al archivo del Centro Integral de Atención al Niño (CIAN) de la Facultad de Odontología, Universidad del Zulia y el Servicio de Odontopediatría de la Maternidad Castillo Plaza, localizados en Maracaibo, estado Zulia, Venezuela. Considerando las características del estudio, se empleó una muestra no probabilística donde se incluyeron 107 radiografías panorámicas pertenecientes a individuos de ambos sexos (56 mujeres y 51 hombres) con edades cronológicas entre los tres y seis años de edad.

Debido a que las radiografías panorámicas fueron obtenidas en diversos centros radiológicos de la región, las mismas se seleccionaron de acuerdo a los siguientes criterios de inclusión, de manera que se pudiera garantizar la calidad de las imágenes: radiografías con contraste y densidad adecuadas, con mínima distorsión, obtenidas de sujetos sin enfermedades sistémicas, talla y peso de acordes a su grupo de edad de acuerdo a la historia clínica, presencia de todos los dientes permanentes (con excepción del tercer molar), sin patologías extensas o anomalías de número, forma, tamaño o posición que pudieran alterar la odontogénesis.

Con fines estadísticos se conformaron cuatro grupos de edad con diferencias de 11 meses entre ellos, constituidos por al menos 10 individuos. La edad real fue calculada restando la fecha de nacimiento de la fecha de la obtención de la radiografía, expresada en años y décimas de años. Las radiografías panorámicas fueron indicadas por razones clínicas, de esta forma no se expuso al sujeto a radiación ionizante adicional para el presente trabajo, siguiendo las directrices de la Declaración de Helsinki (Asociación Médica Mundial, 2017) relacionadas con el estudio en seres humanos, y se garantizó la anonimidad de los pacientes. Los padres o representantes de los individuos firmaron un consentimiento informado para el uso de la información contenida en las historias clínicas con fines docentes o de investigación.

\section{Procedimientos y técnicas}

Registro de los datos. La recolección de los datos personales se realizó a través de las historias clínicas del CIAN y el Servicio de Odontopediatría del Hospital Maternidad Dr. Armando Castillo Plaza, ambos constituyen centros de referencia docenteasistenciales para la región occidental del país. La información se registró en una ficha diseñada para tal fin, y se incluyó: número de historia clínica, nombre y apellido del paciente, nombre de la madre/padre y/o representante, dirección, teléfono, fecha de nacimiento del niño(a), sexo y fecha de la obtención de la de radiografía panorámica.

Digitalización de las imágenes radiográficas. Por razones de distancia y disponibilidad, las radiografías panorámicas seleccionadas considerando los criterios de inclusión, se digitalizaron mediante una cámara fotográfica digital (Fujifilms Will-520, Tokio, Japón) con una resolución de 300 dpi. Las imágenes fueron almacenadas en formato TIFF en un computador y transformadas a escala de grises. Para su análisis se empleó el software Adobe Photoshop, versión CS6 (Adobe System Incorporated, San José, CA, USA), en el que el operador pudo utilizar los recursos de brillo, contraste y magnificación.

Estimación de la edad dental. Las imágenes fueron analizadas por un observador calibrado previa- 
mente, numeradas en forma secuencial, y evaluadas con solo el conocimiento del sexo de individuo. Se asignaron los los estadios de maduración de acuerdo al método de Demirjian et al. a todos los dientes permanentes presentes para conocer el desarrollo dental de los sujetos de la muestra.

Con la finalidad de estimar la ED mediante DEMO, a cada estadio asignado para los siete dientes inferiores izquierdos se le asignó una puntuación de acuerdo a las tablas propuestas por el método, luego se sumaron dichas puntuaciones para obtener el IMD, que después fue convertido en ED empleado las tablas de conversión correspondientes. Para la técnica de siete dientes modificada (Demirjian \& Goldstein), se utilizaron los mismos estadios previamente seleccionados para los siete dientes mandibulares izquierdos y se transformaron en puntuaciones de acuerdo a las tablas modificadas, los valores se sumaron y se obtuvo un IMD que posteriormente fue convertido en ED empleando el percentil 50 de la curva de maduración dental disponible para cada sexo. Para los métodos de cuatro dientes, se utilizaron los mismos estadios de DEMO, estos se transformaron en puntuaciones por medio de las tablas respectivas y el IDM obtenido se convirtió en ED mediante las curvas de maduración disponibles para cada método y sexo.

Para calcular la ED empleando WILL, los estadios asignados previamente mediante DEMO, fueron transformados en puntuaciones empleando las tablas de referencia disponibles para cada sexo y se sumaron para obtener directamente la ED. En relación al método de $\mathrm{CHA}$, se utilizaron los mismos estadios previamente seleccionados mediante DEMO y las tablas de puntuaciones propuestas para obtener el IMD, el cual fue transformado en ED utilizando los valores del percentil 50 de la tabla de conversión disponible por sexo. El observador reevaluó el $15 \%$ de la muestra con un intervalo de 15 días entre el primer análisis, para conocer la confiabilidad de sus observaciones. En este estudio se empleó la nomenclatura de dos dígitos de la Federación Dental Interamericana.

Análisis estadístico. Los datos fueron analizados utilizando el paquete estadístico SPSS versión 15.0 (Statistical Package for the Social Sciences Inc. Chicago, II). Se obtuvieron los estadísticos descriptivos (media y desviación estándar) de las edades cronológicas donde se observaron los diferentes estadios de maduración de acuerdo a los métodos apli- cados. Se calcularon las medias de las edades dentales determinadas por cada método en los grupos de edad para ambos sexos. Se empleó la $T$ de Student para muestras independientes a fin de determinar si existían diferencias entre los sexos en cuanto a las edades cronológicas a las cuales fueron observados los estadios de maduración, así como entre los dientes homólogos de un mismo maxilar y entre dientes antagonistas. Para comparar las diferencias de media entre la EC y la ED calculada mediante los métodos estudiados se aplicó la $T$ de Student para muestras relacionadas. Con la finalidad de estimar la confiabilidad intraobservador, se obtuvo un coeficiente de correlación intra-clase empleando los valores obtenidos para la ED cuando se aplicó WILL. En esta investigación un signo negativo (-) indicó una sobrestimación de la edad. El nivel significancia asumido fue de $p \leq 0,05$.

\section{RESULTADOS}

Con relación al coeficiente de correlación intraclase se obtuvo un valor de $0,93(p<0,001)$, indicando un alto nivel de concordancia entre las evaluaciones realizadas por el evaluador. En las Tablas I a IV se observan las medias de las edades cronológicas en las cuales se evidenciaron los estadios de maduración de acuerdo al método de Demirjian et al. para todos los cuadrantes, en ambos sexos. Al comparar los valores medios obtenidos para los diferentes estadios en los dientes del lado izquierdo y derecho del mismo arco, no se observaron diferencias significativas entre ellos, por lo que el desarrollo dental de los hemiarcos es similar en ambos maxilares. Cuando se compararon los dientes antagonistas, se evidenció una maduración más temprana en los dientes inferiores, solo se observaron diferencias significativas para el estadio $\mathrm{F}$ de los incisivos centrales de ambos lados y el estadio $E$ en los incisivos laterales izquierdos.

En general, las mujeres mostraron una maduración dental más temprana que los hombres. Sin embargo, en el maxilar se observó que los individuos de sexo masculino presentaron valores medios de EC menores en los estadios A, B y C, mientras que los estadios $D$, E y $F$ fueron evidenciados primero en las niñas. Se encontraron diferencias estadísticamente significativas entre los sexos en algunos dientes y estadios (estadio F del 3.6, estadio C de 3.5 y estadio $E$ de 4.6). 
Tabla I. Media de la edad cronológica en la cual se observan los estadios de maduración de Demirjian et al., en el primer cuadrante, ambos sexos.

\begin{tabular}{|c|c|c|c|c|c|c|c|c|}
\hline \multirow{3}{*}{ Diente } & \multirow{3}{*}{ Sexo } & \multicolumn{7}{|c|}{ Estadios de maduración } \\
\hline & & A & B & C & D & $\mathrm{E}$ & $\mathrm{F}$ & G \\
\hline & & Media $( \pm D E)$ & $\operatorname{Media}( \pm D E)$ & Media( $( \pm D E)$ & $\operatorname{Media}( \pm D E)$ & Media $( \pm D E)$ & Media $( \pm D E)$ & $\operatorname{Media}( \pm D E)$ \\
\hline \multirow{2}{*}{17} & Varones & $3,87(0,79)$ & $4,55(1,04)$ & $5,15(0,81)$ & $5,80(1,26)$ & $5,00(-)$ & $6,30(-)$ & - \\
\hline & Hembras & $4,12(0,96)$ & $4,85(0,75)$ & $4,99(1,00)$ & $5,47(0,85)$ & - & - & - \\
\hline \multirow{2}{*}{16} & Varones & - & - & $4,03(0,75)$ & $4,22(0,86)$ & $5,18(0,95)$ & $6,14(0,87)$ & $6,22(0,15)$ \\
\hline & Hembras & - & $5,00(-)$ & $4,15(1,34)$ & $4,30(0,83)$ & $5,32(0,95)$ & $5,31(0,79)$ & $5,85(0,77)$ \\
\hline \multirow{2}{*}{15} & Varones & $4,48(0,78)$ & $4,63(1,21)$ & $4,98(1,07)$ & $6,53(0,32)$ & - & - & - \\
\hline & Hembras & $4,40(1,41)$ & $4,39(0,89)$ & $5,00(0,92)$ & $5,78(0,97)$ & $5,10(-)$ & - & - \\
\hline \multirow{2}{*}{14} & Varones & $4,45(1,25)$ & $4,00(0,82)$ & $4,17(1,13)$ & $4,82(1,07)$ & $6,00(0,92)$ & - & - \\
\hline & Hembras & - & $4,06(1,15)$ & $4,48(0,86)$ & $5,04(0,95)$ & $5,55(0,97)$ & - & - \\
\hline \multirow[b]{2}{*}{13} & Varones & - & - & $3,56(0,75)$ & $5,10(0,93)$ & $5,31(1,02)$ & $6,30(-)$ & - \\
\hline & Hembras & - & - & $4,30(1,12)$ & $4,73(0,83)$ & $5,15(1,03)$ & $6,30(-)$ & - \\
\hline \multirow{2}{*}{12} & Varones & - & - & $4,15(0,94)$ & $4,96(1,10)$ & $5,72(0,87)$ & $6,30(-)$ & - \\
\hline & Hembras & - & - & $4,43(1,00)$ & $4,85(0,99)$ & $5,62(0,96)$ & $5,30(-)$ & - \\
\hline \multirow{2}{*}{11} & Varones & - & - & $3,95(0,76)$ & $4,56(1,18)$ & $5,32(0,83)$ & $6,43(0,15)$ & - \\
\hline & Hembras & - & - & $5,03(0,85)$ & $4,50(1,01)$ & $5,00(0,77)$ & $6,34(0,40)$ & - \\
\hline
\end{tabular}

Tabla II. Media de la edad cronológica en la cual se observan los estadios de maduración de Demirjian et al. en el segundo cuadrante, ambos sexos.

\begin{tabular}{|c|c|c|c|c|c|c|c|c|}
\hline \multirow{3}{*}{ Diente } & \multirow{3}{*}{ Sexo } & \multicolumn{7}{|c|}{ Estadios de maduración } \\
\hline & & A & $\mathrm{B}$ & C & D & $E$ & $\mathrm{~F}$ & $G$ \\
\hline & & Media $( \pm D E)$ & Media( $\pm D E)$ & Media $( \pm D E)$ & Media( $\pm D E)$ & Media( $\pm D E)$ & Media $( \pm D E)$ & Media $( \pm D E)$ \\
\hline \multirow[b]{2}{*}{27} & Varones & $3,94(0,69)$ & $4,51(1,09)$ & $5,01(0,80)$ & $6,10(1,15)$ & $5,40(-)$ & - & - \\
\hline & Hembras & $4,25(1,06)$ & $4,63(0,89)$ & $5,33(0,91)$ & $5,39(0,81)$ & $4,40(-)$ & - & - \\
\hline \multirow{2}{*}{26} & Varones & - & - & $3,85(0,28)$ & $4,17(0,90)$ & $5,02(0,98)$ & $3,94(0,85)$ & $6,22(0,15)$ \\
\hline & Hembras & - & - & $4,30(1,55)$ & $4,16(0,80)$ & $5,35(0,91)$ & $5,12(0,72)$ & $5,83(0,66)$ \\
\hline \multirow{2}{*}{25} & Varones & $4,70(1,13)$ & $4,54(1,10)$ & $4,91(1,07)$ & $6,34(0,35)$ & - & - & - \\
\hline & Hembras & $4,70(1,12)$ & $4,26(0,80)$ & $4,92(0,96)$ & $5,80(0,94)$ & $5,10(-)$ & - & - \\
\hline \multirow{2}{*}{24} & Varones & $4,45(1,20)$ & $3,83(0,92)$ & $4,70(1,09)$ & $4,83(1,07)$ & $6,33(0,31)$ & - & - \\
\hline & Hembras & - & $4,50(1,01)$ & $4,38(0,90)$ & $4,94(0,92)$ & $6,06(0,69)$ & - & - \\
\hline \multirow[b]{2}{*}{23} & Varones & - & - & $3,62(0,80)$ & $5,14(0,86)$ & $5,32(1,05)$ & $6,30(-)$ & - \\
\hline & Hembras & - & - & $4,68(1,40)$ & $4,65(0,81)$ & $5,20(0,94)$ & $6,80(-)$ & - \\
\hline \multirow{2}{*}{22} & Varones & - & - & $4,23(0,88)$ & $4,89(1,14)$ & $5,78(0,83)$ & $6,30(-)$ & - \\
\hline & Hembras & - & - & $4,70(1,03)$ & $4,82(0,92)$ & $5,67(1,05)$ & - & - \\
\hline \multirow{2}{*}{21} & Varones & - & - & $3,95(0,76)$ & $4,57(1,16)$ & $5,34(0,85)$ & $6,43(0,15)$ & - \\
\hline & Hembras & - & - & $5,04(0,95)$ & $4,51(0,99)$ & $5,13(0,81)$ & $5,92(0,93)$ & - \\
\hline
\end{tabular}

Tabla III. Media de la edad cronológica en la cual se observan los estadios de maduración de Demirjian et al. en el tercer cuadrante, ambos sexos.

\begin{tabular}{|c|c|c|c|c|c|c|c|c|}
\hline \multirow{3}{*}{ Diente } & \multirow{3}{*}{ Sexo } & \multicolumn{7}{|c|}{ Estadios de maduración } \\
\hline & & $\begin{array}{c}\text { A } \\
\text { (t) }\end{array}$ & $\begin{array}{c}\text { B } \\
(+D F)\end{array}$ & C & $\begin{array}{c}D \\
(+D F)\end{array}$ & 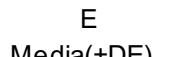 & $\begin{array}{c}F \\
(\mathrm{DF})\end{array}$ & $\begin{array}{c}\text { G } \\
(\mathrm{O} F)\end{array}$ \\
\hline & & Media( $\pm D E)$ & Media( $\pm D E)$ & Media( $\pm D E)$ & Media ( $\pm \mathrm{DE})$ & Media( $( \pm \mathrm{DE})$ & Media $( \pm D E)$ & Media( $\pm D E)$ \\
\hline \multirow{2}{*}{37} & Varones & $4,2(0,86)$ & $4,6(0,78)$ & $5,60(0,75)$ & $5,20(1,15)$ & - & - & - \\
\hline & Hembras & - & - & $5,10(-)$ & $5,36(0,97)$ & $5,08(1,10)$ & $5,02(0,57)$ & $5,62(0,77)$ \\
\hline \multirow{2}{*}{36} & Varones & - & - & $3,40(-)$ & $5,30(0,32)$ & $4,70(0,96)$ & $5,73(0,80)$ & $5,50(0,97)$ \\
\hline & Hembras & - & - & $5,10(-)$ & $3,92(0,74)$ & $5,08(1,10)$ & $5,02(0,57)$ & $5,62(0,77)$ \\
\hline \multirow{2}{*}{35} & Varones & $4,72(0,89)$ & $4,72(1,12)$ & $4,93(0,96)$ & $5,88(0,69)$ & $5,10(1,41)$ & - & - \\
\hline & Hembras & $4,27(0,84)$ & $4,32(0,99)$ & $5,00(0,83)$ & $5,76(0,91)$ & $4,10(-)$ & - & - \\
\hline \multirow{2}{*}{34} & Varones & - & $4,63(0,99)$ & $4,78(1,15)$ & $4,71(0,85)$ & - & - & - \\
\hline & Hembras & $3,40(-)$ & $4,32(0,99)$ & $4,49(1,02)$ & $4,84(0,99)$ & $5,65(0,82)$ & - & - \\
\hline \multirow{2}{*}{33} & Varones & - & - & $3,93(0,74)$ & $4,88(1,07)$ & $5,24(0,81)$ & $5,86(0,83)$ & - \\
\hline & Hembras & - & - & $3,86(0,76)$ & $4,37(0,92)$ & $5,23(0,86)$ & $5,96(0,73)$ & - \\
\hline \multirow[b]{2}{*}{32} & Varones & - & - & $4,18(0,85)$ & $4,90(1,05)$ & $4,87(1,02)$ & $6,17(0,22)$ & - \\
\hline & Hembras & - & - & $4,17(0,82)$ & $4,71(0,89)$ & $4,99(1,10)$ & $5,93(0,40)$ & $6,80(-)$ \\
\hline \multirow{2}{*}{31} & Varones & - & - & $4,30(0,94)$ & $4,50(1,12)$ & $5,18(0,67)$ & $5,20(1,08)$ & $6,20(0,21)$ \\
\hline & Hembras & - & - & $4,34(1,08)$ & $4,37(0,92)$ & $4,87(1,02)$ & $5,44(0,74)$ & $6,05(0,49)$ \\
\hline
\end{tabular}


Tabla IV. Media de la edad cronológica en la cual se observan los estadios de maduración de Demirjian et al., en el cuarto cuadrante, ambos sexos.

\begin{tabular}{|c|c|c|c|c|c|c|c|c|}
\hline \multirow{3}{*}{ Diente } & \multirow{3}{*}{ Sexo } & \multicolumn{7}{|c|}{ Estadios } \\
\hline & & A & B & C & D & E & $\mathrm{F}$ & G \\
\hline & & Media( $\pm D E)$ & Media( $( \pm D E)$ & $\operatorname{Media}( \pm D E)$ & Media( $\pm D E)$ & Media ( $\pm D E)$ & Media( $( \pm D E)$ & Media( $\pm D E)$ \\
\hline \multirow{2}{*}{47} & Varones & $3,98(0,68)$ & $4,68(1,10)$ & $5,41(0,85)$ & $5,29(1,13)$ & - & - & - \\
\hline & Hembras & $4,06(1,03)$ & $4,69(0,89)$ & $4,99(0,89)$ & $5,57(0,81)$ & - & - & - \\
\hline \multirow[b]{2}{*}{46} & Varones & - & $3,60(-)$ & - & $4,06(0,59)$ & $4,98(0,85)$ & $5,46(1,26)$ & $5,72(0,91)$ \\
\hline & Hembras & $4,00(-)$ & - & $5,10(-)$ & $3,80(0,73)$ & $5,21(0,89)$ & $5,00(0,66)$ & $5,63(0,73)$ \\
\hline \multirow{2}{*}{45} & Varones & $4,58(0,87)$ & $4,80(1,03)$ & $5,16(1,03)$ & $6,36(0,25)$ & $4,10(-)$ & - & - \\
\hline & Hembras & $4,37(1,03)$ & $4,63(1,00)$ & $5,00(0,83)$ & $5,25(1,19)$ & $4,00(-)$ & - & - \\
\hline \multirow[b]{2}{*}{44} & Varones & $3,60(-)$ & $4,41(0,72)$ & $4,85(1,08)$ & $4,45(1,11)$ & $6,26(0,49)$ & - & - \\
\hline & Hembras & - & $4,16(1,15)$ & $4,17(0,91)$ & $4,90(0,91)$ & $5,46(0,87)$ & - & - \\
\hline \multirow{2}{*}{43} & Varones & - & - & $3,90(0,79)$ & $4,74(1,23)$ & $5,22(0,76)$ & $6,66(0,55)$ & - \\
\hline & Hembras & - & - & $3,52(0,33)$ & $4,76(1,09)$ & $5,01(0,88)$ & $5,85(1,07)$ & - \\
\hline \multirow[b]{2}{*}{42} & Varones & - & - & $4,68(1,17)$ & $4,51(1,29)$ & $5,11(0,64)$ & $5,20(1,08)$ & $6,25(0,21)$ \\
\hline & Hembras & - & - & $4,16(0,90)$ & $4,75(0,86)$ & $5,02(1,08)$ & $5,77(0,45)$ & $6,80(-)$ \\
\hline \multirow{2}{*}{41} & Varones & - & - & $4,30(0,94)$ & $4,72(1,18)$ & $4,90(0,84)$ & $6,01(1,05)$ & - \\
\hline & Hembras & - & - & $4,30(1,08)$ & $4,37(0,92)$ & $4,87(1,02)$ & $5,44(0,72)$ & $6,05(0,49)$ \\
\hline
\end{tabular}

En la Tabla $V$ se presentan las medias y las diferencias de media entre la EC y la ED calculada por cada método. En el total de la muestra se observó la sobre-estimación de la edad para todos los métodos, siendo la diferencia estadísticamente significativa. En los hombres la sobre-estimación fue menor para $\mathrm{CHA}$, seguido de WILL, DEM4AL, DEM7AL, DEM4 y DEMO, mientras que en las mujeres dicha sobre-estimación fue menor en WILL seguido de CHAI, DEM7AL, DEM4AL, DEM4 y DEMO.

Tabla V. Medias y diferencia de media entre la edad cronológica y la edad dental estimada por los diferentes métodos estudiados.

\begin{tabular}{|c|c|c|c|c|c|c|c|}
\hline \multirow{2}{*}{ Sexo } & \multirow{2}{*}{$\mathrm{EC}(\mathrm{DE})$} & \multirow{2}{*}{ Método } & \multirow{2}{*}{$\mathrm{ED}(\mathrm{DE})$} & \multirow{2}{*}{$\mathrm{EC}-\mathrm{ED}(\mathrm{DE})$} & \multicolumn{2}{|c|}{$\mathrm{IC}(95 \%)$} & \multirow{2}{*}{ Sig. } \\
\hline & & & & & Min. & Max. & \\
\hline \multirow{6}{*}{ Varones } & \multirow{6}{*}{$5,92(1,02)$} & DEMO & $5,83(1,38)$ & $-0,91(1,33)$ & $-1,29$ & $-0,54$ & $0,000^{\star *}$ \\
\hline & & DEM7AL & $5,47(1,41)$ & $-0,55(1,36)$ & $-0,94$ & $-0,17$ & $0,005^{*}$ \\
\hline & & DEM4 & $5,33(1,45)$ & $-0,61(1,44)$ & $-1,02$ & $-0,21$ & $0,004^{*}$ \\
\hline & & DEM4AL & $5,40(1,44)$ & $-0,48(1,45)$ & $-0,89$ & $-0,07$ & $0,020^{*}$ \\
\hline & & WILL & $5,29(1,23)$ & $-0,36(1,09)$ & $-0,67$ & $-0,06$ & $0,020^{*}$ \\
\hline & & $\mathrm{CHA}$ & $5,17(1,18)$ & $-0,25(1,12)$ & $-0,57$ & $-0,06$ & 0,111 \\
\hline \multirow{6}{*}{ Hembras } & \multirow{6}{*}{$4,89(1,00)$} & DEMO & $5,79(1,37)$ & $-0,89(1,17)$ & $-1,21$ & $-0,58$ & $0,000^{\star *}$ \\
\hline & & DEM7AL & $5,50(1,20)$ & $-0,60(1,06)$ & $-0,89$ & $-0,32$ & $0,000^{* *}$ \\
\hline & & DEM4 & $5,75(1,37)$ & $-0,86(1,20)$ & $-1,18$ & $-0,54$ & $0,000^{\star *}$ \\
\hline & & DEM4AL & $5,61(1,30)$ & $-0,71(1,23)$ & $-1,04$ & $-0,38$ & $0,000^{* *}$ \\
\hline & & WILL & $5,15(1,24)$ & $-0,25(1,09)$ & $-0,55$ & 0,03 & 0,082 \\
\hline & & $\mathrm{CHA}$ & $5,21(1,06)$ & $-0,31(0,94)$ & $-0,56$ & $-0,06$ & $0,015^{*}$ \\
\hline \multirow{6}{*}{ Total } & \multirow{6}{*}{$4,90(1,00)$} & DEMO & $5,81(1,37)$ & $-0,90(1,24)$ & $-1,14$ & $-0,66$ & $0,000^{\star \star}$ \\
\hline & & DEM7AL & $5,49(1,29)$ & $-0,58(1,21)$ & $-0,31$ & $-0,35$ & $0,000^{* *}$ \\
\hline & & DEM4 & $5,65(1,41)$ & $-0,74(1,32)$ & $-1,00$ & $-0,49$ & $0,000^{* *}$ \\
\hline & & DEM4AL & $5,51(1,40)$ & $-0,60(1,34)$ & $-0,86$ & $-0,35$ & $0,000^{* *}$ \\
\hline & & WILL & $5,21(1,23)$ & $-0,31(1,09)$ & $-0,52$ & $-0,10$ & $0,004^{*}$ \\
\hline & & $\mathrm{CHA}$ & $4,08(0,80)$ & $-0,01(0,95)$ & $-0,71$ & 0,71 & 0,959 \\
\hline
\end{tabular}

Prueba t para muestras relacionadas. Significancia $p<0,05$; * Significancia $p<0,000$. EC: edad cronológica; DEMO: método de Demirjian et al. siete dientes original; DEM7Al: método de Demirjian et al. siete dientes alternativo; DEM4: método de Demirjian et al. cuatro dientes; DEM4AI: método de Demirjian et al. cuatro dientes alternativo; WILL: método de Willems et al.; CHA: método de Chaillet et al.; ED: edad dental; DE: desviación estándar; IC: intervalo de confianza; Max.: máximo; Min.: mínimo; Sig: significancia. 
Tabla VI. Medias y diferencia de media entre la edad cronológica y la edad dental estimada por los métodos estudiados, sexo masculino.

\begin{tabular}{|c|c|c|c|c|c|c|c|}
\hline \multirow{2}{*}{$\begin{array}{c}\text { Grupo de } \\
\text { edad }\end{array}$} & \multirow{2}{*}{$\mathrm{EC}(\mathrm{DE})$} & \multirow{2}{*}{ Método } & \multirow{2}{*}{$\mathrm{ED}(\mathrm{DE})$} & \multirow{2}{*}{ EC-ED(DE) } & \multicolumn{2}{|c|}{$\mathrm{IC}(95 \%)$} & \multirow{2}{*}{ Sig } \\
\hline & & & & & Min. & Max. & \\
\hline \multirow{6}{*}{3} & \multirow{6}{*}{$3,37(0,25)$} & DEMO & $4,60(1,55)$ & $-1,22(1,59)$ & $-2,45$ & 0,05 & $0,051^{*}$ \\
\hline & & DEM7AL & $4,32(1,38)$ & $-0,94(1,40)$ & $-2,02$ & 0,13 & $0,079^{*}$ \\
\hline & & DEM4 & $4,73(1,49)$ & $-1,35(1,54)$ & $-2,54$ & $-0,17$ & $0,030^{*}$ \\
\hline & & DEM4AL & $4,52(1,51)$ & $-1,14(1,54)$ & $-2,33$ & 0,04 & $0,057^{*}$ \\
\hline & & WILL & $4,15(0,73)$ & $-0,77(0,86)$ & $-1,44$ & $-0,11$ & $0,027^{*}$ \\
\hline & & $\mathrm{CHA}$ & $4,02(0,79)$ & $-0,65(0,92)$ & $-1,36$ & 0,06 & 0,063 \\
\hline \multirow{6}{*}{4} & \multirow{6}{*}{$4,49(0,35)$} & DEMO & $5,79(1,29)$ & $-1,30(1,47)$ & $-2,05$ & $-0,54$ & $0,002^{*}$ \\
\hline & & DEM7AL & $5,37(1,25)$ & $-0,88(1,43)$ & $-1,61$ & $-0,14$ & $0,022^{*}$ \\
\hline & & DEM4 & $5,21(1,33)$ & $-0,71(1,50)$ & $-1,49$ & 0,05 & 0,068 \\
\hline & & DEM4AL & $5,17(1,38)$ & $-0,68(1,57)$ & $-1,49$ & 0,12 & 0,093 \\
\hline & & WILL & $5,09(1,08)$ & $-0,59(1,23)$ & $-1,23$ & 0,03 & 0,064 \\
\hline & & $\mathrm{CHA}$ & $5,08(1,08)$ & $0,70(0,28)$ & 0,56 & 0,84 & $0,000^{* *}$ \\
\hline \multirow{6}{*}{5} & \multirow{6}{*}{$5,35(0,28)$} & DEMO & $5,87(1,04)$ & $-0,52(1,05)$ & $-1,13$ & 0,08 & 0,088 \\
\hline & & DEM7AL & $5,57(1,08)$ & $-0,22(1,03)$ & $-0,83$ & 0,39 & 0,448 \\
\hline & & DEM4 & $5,60(0,98)$ & $-0,25(0,97)$ & $-0,82$ & 0,30 & 0,342 \\
\hline & & DEM4AL & $5,50(1,01)$ & $-0,15(1,00)$ & $-0,72$ & 0,42 & 0,585 \\
\hline & & WILL & $5,27(0,86)$ & $0,07(0,79)$ & $-0,36$ & 0,53 & 0,733 \\
\hline & & $\mathrm{CHA}$ & $5,19(0,96)$ & $0,15(0,92)$ & $-0,37$ & 0,68 & 0,544 \\
\hline \multirow{6}{*}{6} & \multirow{6}{*}{$6,30(0,26)$} & DEMO & $6,87(0,98)$ & $-0,57(1,10)$ & $-1,31$ & 0,16 & 0,116 \\
\hline & & DEM7AL & $6,46(1,42)$ & $-0,16(1,52)$ & $-1,18$ & 0,86 & 0,729 \\
\hline & & DEM4 & $6,61(1,60)$ & $-0,31(1,70)$ & $-1,46$ & 0,82 & 0,549 \\
\hline & & DEM4AL & $6,38(1,52)$ & $-0,08(1,62)$ & $-1,17$ & 1,00 & 0,871 \\
\hline & & WILL & $6,54(1,70)$ & $-0,24(1,27)$ & $-1,10$ & 0,69 & 0,537 \\
\hline & & $\mathrm{CHA}$ & $6,22(0,98)$ & $0,07(1,10)$ & $-0,67$ & 0,81 & 0,832 \\
\hline
\end{tabular}

Prueba t para muestras relacionadas. Significancia $p<0,05$; ${ }^{* *}$ Significancia $p<0,000$. EC: edad cronológica; DEMO: método de Demirjian et al. siete dientes original; DEM7Al: método de Demirjian et al. siete dientes alternativo; DEM4: método de Demirjian et al. cuatro dientes; DEM4Al: método de Demirjian et al. cuatro dientes alternativo; WILL: método de Willems et al.; CHA: método de Chaillet et al.; ED: edad dental; DE: desviación estándar; IC: intervalo de confianza; Max.: máximo; Min.: mínimo; Sig: significancia.

Cuando se estudiaron los grupos de edad, se verificó para los hombres (Tabla VI) que en general, la edad estimada por los métodos estudiados fue sobre-estimada, con excepción de CHA para los grupos de edad de cuatro a seis años, este método mostró la menor diferencia EC-ED para los grupos de tres y seis años, mientras que WILL fue más preciso en los grupos de cuatro y cinco años. DEMO mostró la mayor diferencia EC-ED en todos los grupos de edad.

En las mujeres (Tabla VII), la edad fue sobreestimada por los métodos estudiados en los grupos de tres a cinco años, mientras que en el grupo de seis años se observó en general una sub-estimación. WILL mostró la menor diferencia EC-ED en los grupos de tres, cinco y seis años, en cuanto que en el grupo de cuatro años presentó una diferencia EC-
ED similar a CHA. En los grupos de edad de tres a cinco años DEMO mostró la menor precisión, mientras que en el grupo de seis años se verificó que CHA tuvo la mayor diferencia EC-ED.

Se construyeron gráficos para representar la relación entre la media de la EC y la ED estimada por los métodos en ambos sexos. En la Figura $1 \mathrm{se}$ evidencian los resultados obtenidos para el método de Demirjian y sus modificaciones; en ambos sexos se observó una cercanía de las edades dentales estimadas a la EC en los grupos de cinco y seis años de edad. Para WILL (Fig. 2) se observó la sobreestimación de la edad en los grupos de tres a cinco años en ambos sexos, mientas que CHA (Fig. 3) mostró la sobre-estimación de la edad en los grupo de tres y cuatro años tanto en las mujeres como en los hombres. 
CARRIZO, A. L.; QUEVEDO-PIÑA, M. L. \& ORTEGA-PERTUZ, A. I. Maduración de dientes permanentes en niños venezolanos entre 3 y 6 años: aplicabilidad en la estimación de la edad dental con fines forenses. Int. J. Odontostomat., 14(3):430-441, 2020.

Tabla VII. Medias y diferencia de media entre la edad cronológica y la edad dental estimada por los métodos estudiados, sexo femenino.

\begin{tabular}{|c|c|c|c|c|c|c|c|}
\hline \multirow{2}{*}{$\begin{array}{c}\text { Grupo de } \\
\text { edad }\end{array}$} & \multirow{2}{*}{$\mathrm{EC}(\mathrm{DE})$} & \multirow{2}{*}{ Método } & \multirow{2}{*}{$\mathrm{ED}(\mathrm{DE})$} & \multirow{2}{*}{ EC-ED(DE) } & \multicolumn{2}{|c|}{$\mathrm{IC}(95 \%)$} & \multirow{2}{*}{ Sig. } \\
\hline & & & & & Min. & Max. & \\
\hline \multirow{6}{*}{3} & \multirow{6}{*}{$3,33(0,18)$} & DEMO & $4,52(1,26)$ & $-1,18(1,23)$ & $-2,13$ & $-0,23$ & $0,020^{*}$ \\
\hline & & DEM7AL & $4,38(1,00)$ & $-1,05(0,99)$ & $-1,82$ & $-0,28$ & $0,013^{*}$ \\
\hline & & DEM4 & $4,37(1,01)$ & $-1,04(1,03)$ & $-1,84$ & $-0,24$ & $0,017^{*}$ \\
\hline & & DEM4AL & $4,30(1,22)$ & $-0,96(1,24)$ & $-1,92$ & $-0,01$ & $0,048^{*}$ \\
\hline & & WILL & $3,91(0,86)$ & $-0,57(0,85)$ & $-1,23$ & 0,08 & 0,678 \\
\hline & & $\mathrm{CHA}$ & $4,05(1,12)$ & $-0,72(1,10)$ & $-1,57$ & 0,12 & 0,085 \\
\hline \multirow{6}{*}{4} & \multirow{6}{*}{$4,40(0,29)$} & DEMO & $5,63(1,25)$ & $-1,23(1,16)$ & $-1,81$ & $-0,65$ & $0,000^{* *}$ \\
\hline & & DEM7AL & $5,41(1,07)$ & $-1,01(0,98)$ & $-1,50$ & $-0,32$ & $0,000^{* *}$ \\
\hline & & DEM4 & $5,76(1,22)$ & $-1,36(1,14)$ & $-1,90$ & $-0,79$ & $0,000^{* *}$ \\
\hline & & DEM4AL & $5,61(1,20)$ & $-1,21(1,11)$ & $-1,77$ & $-0,66$ & $0,000^{* *}$ \\
\hline & & WILL & $5,08(1,13)$ & $-0,68(1,00)$ & $-1,18$ & $-0,18$ & 0,010 \\
\hline & & $\mathrm{CHA}$ & $5,09(0,90)$ & $-0,69(0,79)$ & $-1,09$ & $-0,30$ & $0,002^{*}$ \\
\hline \multirow{6}{*}{5} & \multirow{6}{*}{$5,32(0,26)$} & DEMO & $6,05(1,37)$ & $-0,72(1,29)$ & $-1,34$ & $-0,10$ & $0,025^{*}$ \\
\hline & & DEM7AL & $5,66(1,21)$ & $-0,34(1,14)$ & $-0,89$ & 0,20 & 0,208 \\
\hline & & DEM4 & $5,96(1,35)$ & $-0,63(1,29)$ & $-1,26$ & $-0,01$ & 0,460 \\
\hline & & DEM4AL & $5,82(1,29)$ & $-0,50(1,25)$ & $-1,10$ & 0,10 & 0,099 \\
\hline & & WILL & $5,41(1,15)$ & $-0,09(1,10)$ & $-0,62$ & 0,44 & 0,727 \\
\hline & & $\mathrm{CHA}$ & $5,52(0,89)$ & $-0,20(0,83)$ & $-0,60$ & 0,20 & 0,311 \\
\hline \multirow{6}{*}{6} & \multirow{6}{*}{$6,37(0,29)$} & DEMO & $6,73(0,77)$ & $-0,36(0,67)$ & $-0,82$ & 0,10 & 0,113 \\
\hline & & DEM7AL & $6,35(0,82)$ & $0,02(0,67)$ & $-0,46$ & 0,50 & 0,926 \\
\hline & & DEM4 & $6,61(1,14)$ & $-0,24(1,03)$ & $-0,97$ & 0,49 & 0,480 \\
\hline & & DEM4AL & $6,38(1,25)$ & $0,01(1,09)$ & $-0,79$ & 0,77 & 0,978 \\
\hline & & WILL & $5,90(1,17)$ & $0,46(1,09)$ & $-0,31$ & 1,25 & 0,210 \\
\hline & & $\mathrm{CHA}$ & $5,87(0,83)$ & $0,52(0,69)$ & 1,01 & 1,01 & $0,043^{*}$ \\
\hline
\end{tabular}

Prueba t para muestras relacionadas. Significancia $p<0,05$; ${ }^{*}$ Significancia $p<0,000$. EC: edad cronológica; DEMO: método de Demirjian et al. siete dientes original; DEM7AI: método de Demirjian et al. siete dientes alternativo; DEM4: método de Demirjian et al. cuatro dientes; DEM4AI: método de Demirjian et al. cuatro dientes alternativo; WILL: método de Willems et al.; CHA: método de Chaillet et al.; ED: edad dental; DE: desviación estándar; IC: intervalo de confianza; Max.: máximo; Min.: mínimo; Sig: significancia.
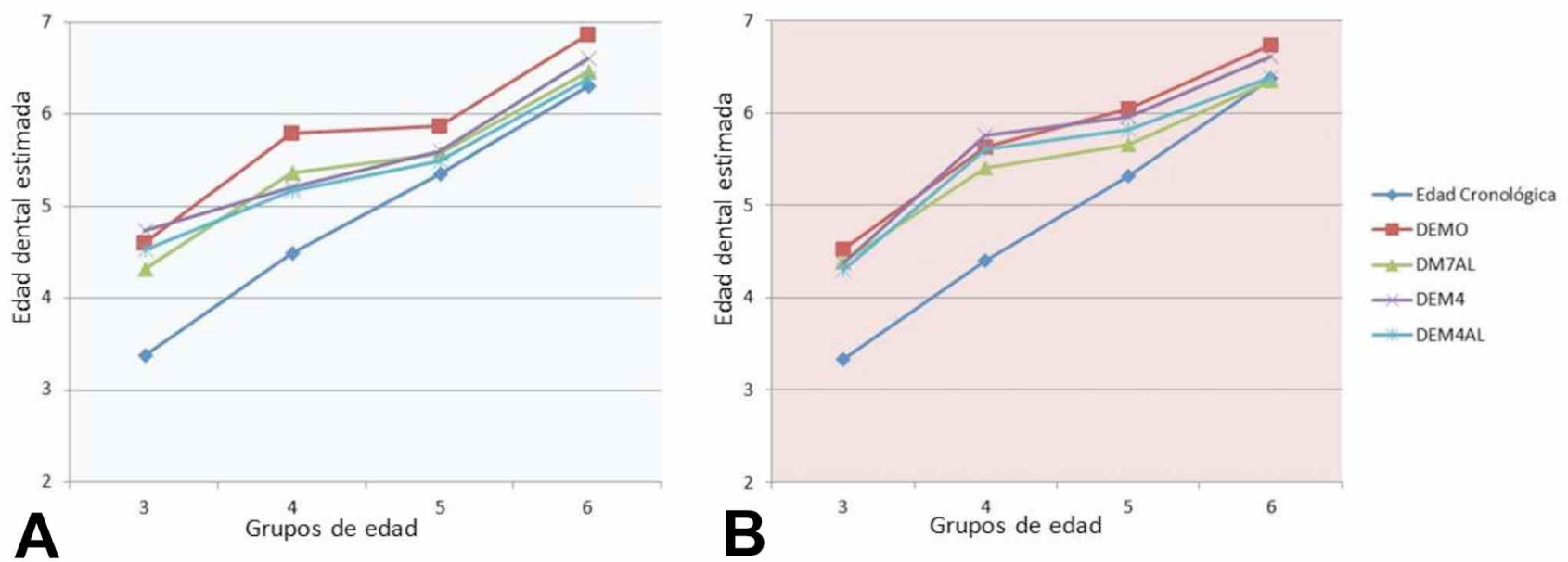

Fig. 1. Relación entre la edad cronológica y la edad dental estimada por el método de Demirjian et al. y sus modificaciones. A: Masculino; B: Femenino. DEMO: método de Demirjian et al. siete dientes original; DEM7Al: método de Demirjian et al. siete dientes alternativo; DEM4: método de Demirjian et al. cuatro dientes; DEM4Al: método de Demirjian et al. cuatro dientes alternativo; WILL: método de Willems et al.; CHA: método de Chaillet et al. 

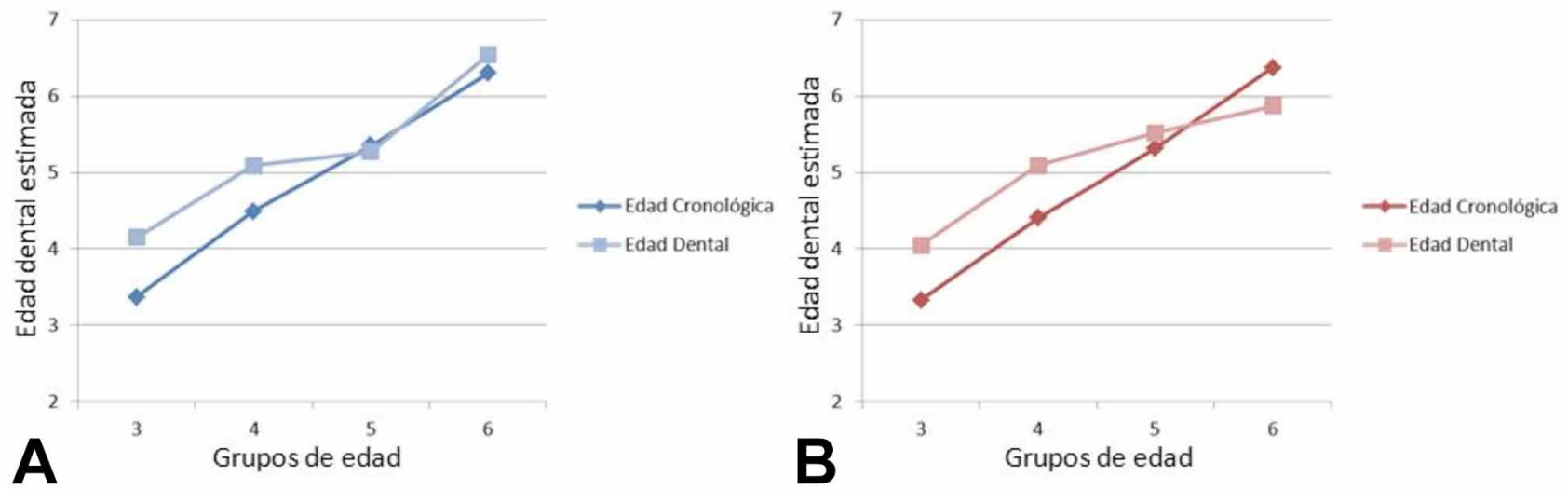

Fig. 2. Relación entre la edad cronológica y la edad dental estimada por el método de Willems et al. A: Masculino; B: Femenino
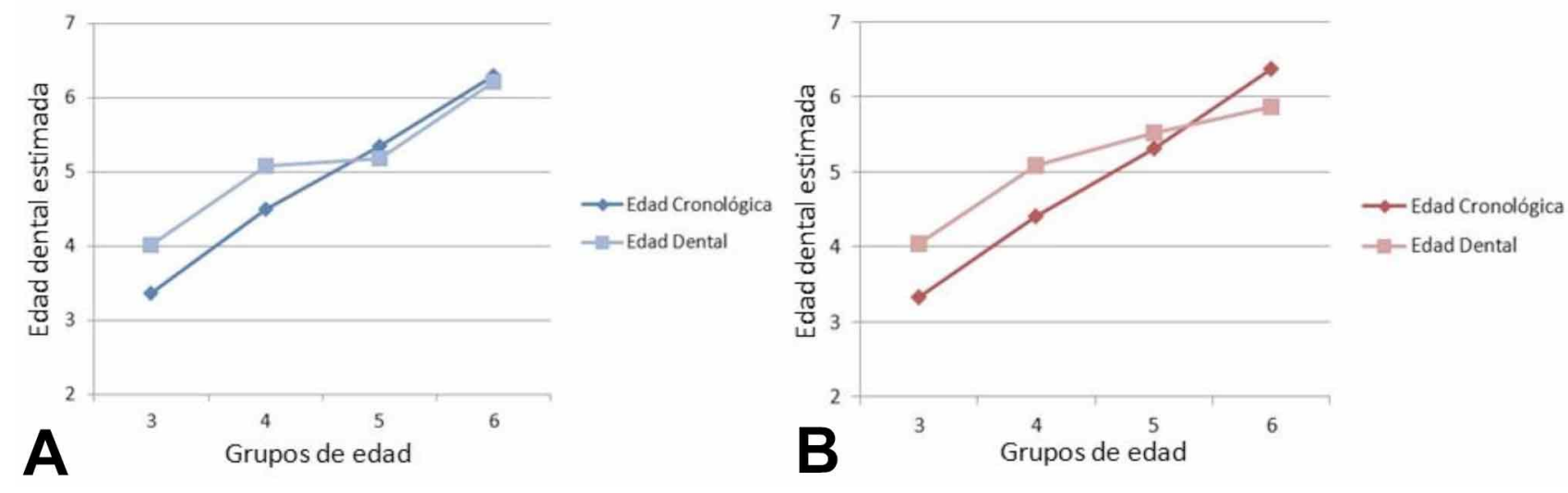

Fig. 3. Relación entre la edad cronológica y la edad dental estimada por el método de Chaillet et al. A: Masculino; B: Femenino.

\section{DISCUSIÓN}

La ED ha sido empleada en la práctica odontológica para evaluar si la maduración de la dentición del paciente se encuentra dentro del promedio para su grupo de edad; así como en la ciencia forense para la estimación de la edad en individuos fallecidos o vivos sin documentos válidos de identificación (CruzLanderia et al.; Nik-Hussein et al.; Urzel \& Bruzek; Medina \& Blanco; Onat Altan et al; Mohammed et al.). En este estudio se emplearon radiografías panorámicas de pacientes con edades comprendidas entre los tres y seis años, en ellas se observa la dentición completa en una sola imagen, lo que proporciona a los investigadores una manera única y efectiva de evaluar el desarrollo dental (Nik-Hussein et al.; Nur et al.; Galic et al.).
Entre los diversos métodos diseñados para la estimación de la ED mediante la observación de la mineralización dental en imágenes radiográficas se encuentran los de Demirjian et al., Demirjian \& Goldstein, Willems et al. y Chaillet et al. Debido a su enfoque morfológico, la valoración del desarrollo de la dentición se basa principalmente en el estudio de secuencias predecibles de estadios de maduración y no en función de dimensiones, lo que ha permitido obtener resultados reproducibles (Nik-Hussein et al.; Urzel \& Bruzek; Onat Altan et al.; Mohammed et al.).

Son limitados los estudios en los que se incluya la cronología del desarrollo de todos los dientes permanentes Asimismo, es importante mencio- 
nar las dificultades inherentes a la inclusión de pacientes tan jóvenes, ya sea por razones técnicas, legales o éticas (Lee et al.) En el rango de edad estudiado en el presente trabajo es inherente la sobreposición de la imagen de los elementos dentarios temporarios y los permanentes en desarrollo en la radiografía panorámica, lo que en ocasiones dificultó la evaluación o la inclusión de la radiografía dentro de la muestra.

En esta investigación se obtuvieron las edades cronológicas medias a las cuales fueron observados los estadios de maduración de la dentición permanente (con excepción del tercer molar), y se encontró que en general, dichos estadios fueron alcanzados en edades más tempranas por las mujeres, lo cual ha sido descrito en estudios previos (NikHussein et al.; Ambarkova et al.; Aissaoui et al.) aunque no se observó una diferencia significativa entre los sexos. Se observó además, un avance en la maduración de los dientes inferiores en comparación con los superiores, lo que puede estar relacionado con el proceso de estimulación propia de la mandíbula mediante las capacidades funcionales de la masticación, fonación y crecimiento del macizo facial (Águila \& Enlow; Gardner et al.).

La precisión de la estimación de la ED ha sido definida como la proximidad con que la EC del individuo puede ser calculada y es el resultado de la diferencia EC-ED, siendo q esa proximidad entre ambas edades lo que define la aplicabilidad del método (Willems et al.; Nur et al.; Aissaoui et al.). En la muestra estudiada, los resultados obtenidos concuerdan con investigaciones previas, en donde los individuos menores a los seis años presentan una ED avanzada con respecto a la EC (Nik-Hussein et al.; Nur et al.; Flood et al., 2013; Urzel \& Bruzek; Ambarkova et al.; Aissaoui et al.; Onat Altan et al.). Independientemente del sexo, CHA fue más preciso, sin embargo, al investigar los valores de la diferencia EC-ED en los hombres y mujeres por separado, WILL mostró una mejor precisión en las niñas, lo que ha sido descrito por otros autores (Liversidge et al.; Nik-Hussein et al.; Djuvik et al.; Urzel \& Bruzek; Ambarkkova et al.; Mohammed et al.; Onat Altan et al.), mientras que CHA fue más preciso en los niños, tal como lo señalan Cruz-Landeira et al. y Urzel \& Bruzek.

El método de Demirjian et al. original presentó la mayor diferencia EC-ED, lo que fue reportado por Flood et al. y Ambarkova et al. cuando compara- ron las distintas versiones del método. La sobre-estimación de la edad encontrada refleja el avance en la maduración dental de los sujetos de la presente investigación con respecto a aquellos empleados en la estandarización de los métodos, siendo que la mayor sobre-estimación encontrada para DEMO era esperada debido a la diferencia de casi treinta años entre la publicación del mismo y la propuestas de Willems et al. y Chaillet et al., lo que pudiera expresar una tendencia secular hacia una maduración más temprana.

Ahora bien, se ha afirmado que para la antropología forense un rango aceptable de diferencia ECED se encuentra entre $\pm 0,5 \mathrm{a} \pm 1$ años (Onat Altan et al.; Ambarkova et al.), en esta investigación, cuando las diferencias fueron estudiadas por grupos de edad, se encontró que para los grupos de tres y cuatro años, el método de Demirjian et al. y Demirjian \& Goldstein superó este rango, lo que fue particularmente notorio en las mujeres.

Considerando otros estudios realizados en venezolanos, Cruz-Landeira et al. observaron una sobre-estimación de la edad en niños de ambos sexos menores de ocho años al aplicar el método de Demirjian et al. en merideños (Medina \& Blanco), lo cual coincide con lo evidenciando en esta investigación. En oposición a lo observado en sujetos del área metropolitana de Caracas, en los cuales Medina \& Blanco reportaron una sub-estimación de la edad en los grupos de tres a seis años, utilizando los métodos de Demirjian \& Goldstein y Willems et al. Hasta el presente no se conocen trabajos realizados en zulianos menores de seis años de edad.

Las diferencias encontradas entre la ED estimada por los métodos empleados y la EC de la muestra, puede estar relacionada con discrepancias entre los por factores ambientales, genética, estatus socio-económico, nutrición, hábitos dietéticos y estilos de vida entre los sujetos en estudio y aquello empleados para la construcción de las referencias de los métodos. Por otro lado se ha señalado que dichas diferencias también están asociadas al tamaño de la muestra, el rango de edad y el enfoque estadístico empleado (Chaillet et al.; Liversidege et al.; Urzel \& Bruzek). Cuando se evalúan las diferencias entre sub-grupos de venezolanos, las mismas son atribuidas también al mestizaje de amerindios, españoles y afrodescendientes que se produjo en distinta proporción en las áreas geográficas del país (Rodríguez-Larralde et al., 2001). 


\section{CONCLUSIONES}

Entre los tres y seis años, las mujeres muestran un desarrollo dental más avanzado que los hombres. La maduración de los dientes permanentes es más temprana en la mandíbula, sin embargo, no existen diferencias significativas entre dientes homólogos de un mismo arco o entre antagonistas. Las edades estimadas por los métodos de Demirjian et al., Demirjian \& Goldstein, Willems et al., y Chaillet et al. fueron sobre-estimadas. En cuanto a la precisión, en términos de una diferencia entre la edad cronológica del individuo y la edad dental calculada, el método de Chaillet et al. fue más preciso para el total de la muestra. Al investigar los resultados para hombres y mujeres por separado, el método de Willems et al. mostró una mejor precisión en las niñas y el de Challet et al. en los niños, mientras que el método de Dermijian et al. (1973) original presentó la mayor diferencia para ambos sexos, lo que fue notorio en los grupos de tres y cuatro años de edad.

CARRIZO, A. L.; QUEVEDO-PIÑA, M. L. \& ORTEGAPERTUZ, A. I. Permanent teeth maturation in 3-6 years old Venezuelan children: applicability in dental age estimation with forensic purposes. Int. J. Odontostomat., 14(3):430-441, 2020.

ABSTRACT: The objective of this work was to study the maturation of permanent teeth in Venezuelan children between three and six years, and its applicability in the estimation of dental age (DA) for forensic purposes. A descriptive, retrospective, cross-sectional and field study was carried out. The sample consisted of 107 panoramic radiographs of Venezuelan individuals ( 56 females and 51 males). The stages of maturation were assigned to the permanent teeth of the four quadrants according to the original Demirjian et al. (1973) method, while the DA was calculated by Demirjian's et al. method and its modifications (Demirjian \& Goldstein, 1976), Willems et al. (2001) and Chaillet et al. (2005). It was found that females reached the maturation stages at younger ages than males. The overestimation of the chronological age (CA) by all methods was observed in the total sample, being the method of Chaillet et al., the most accurate. When studying the sexes separately, the method of Chaillet et al., presented the lowest CA-DA difference for males and Chaillet et al., in females. The method of Demirjian et al., showed the greatest CA-DA difference for both sexes. The methods of Chaillet et al. and Willems et al. are applicable for age determination purposes in the studied sample, its utility being sex dependent.

KEY WORDS: forensic dentistry, dental maturation, dental age, age estimation by teeth.

\section{REFERENCIAS BIBLIOGRÁFICAS}

Águila, J. F. \& Enlow, D. H. Crecimiento Cráneofacial, Ortodoncia y Ortopedia. $2^{\mathrm{a}}$ ed. Caracas, Actualidades Médico Odontológicas Latinoamérica, 1993.

Aissaoui, A.; Salem, N. H.; Mougou, M.; Maatouk, F. \& Chadly, A. Dental age assessment among Tunisian children using the Demirjian method. J. Forensic Dent. Sci., 8(1):47-51, 2016.

Ambarkova, V.; Galic, I.; Vodanovic, M.; Biocina-Lukenda, D. \& Brkic', H. Dental age estimation using Demirjian and Willems methods: cross sectional study on children from the Former Yugoslav Republic of Macedonia. Forensic Sci. Int., 234:187.e1-7, 2014.

Asociación Médica Mundial. Declaración de Helsinki. Principios Éticos para las Investigaciones Médicas en Seres Humanos. Ferney-Voltaire, Asociación Médica Mundial, 2017. Disponible en: https://www.wma.net/es/policies-post/declaracion-dehelsinki-de-la-amm-principios-eticos-para-las-investigacionesmedicas-en-seres-humanos/

Chaillet, N.; Nyström, M. \& Demirjian, A. Comparison of dental maturity in children of different ethnic origins: international maturity curves for clinicians. J. Forensic Sci., 50(5):1164-74, 2005.

Cruz-Landeira, A.; Linares-Argote, J.; Martínez-Rodríguez, M.; Rodríguez-Calvo, M. S.; Otero, X. L. \& Concheiro, L. Dental age estimation in Spanish and Venezuelan children. Comparison of Demirjian and Chaillet's scores. Int. J. Legal Med., 124(2):105-12, 2010.

Demirjian, A. \& Goldstein, H. New systems for dental maturity based on seven and four teeth. Ann. Hum. Biol., 3(5):411-21, 1976.

Demirjian, A.; Goldstein, H. \& Tanner, J. M. A new system of dental age assessment. Hum. Biol., 45(2):211-27, 1973.

Djukic, K.; Zelic, K.; Milenkovic, P.; Nedeljkovic, N. \& Djuric, M. Dental age assessment validity of radiographic methods on Serbian children population. Forensic Sci. Int., 231(1-3):398.e1-5, 2013.

Flood, S. J.; Franklin, D.; Turlach, B. A. \& McGeachie, J. A comparison of Demirjian's four dental development methods for forensic age estimation in South Australian sub-adults. J. Forensic Leg. Med., 20(7):875-83, 2013.

Galic, I.; Vodanovic, M.; Jankovic, S.; Mihanovic, F.; Nakas, E.; Prohic, S.; Galic, E. \& Brkic, H. Dental age estimation on BosnianHerzegovinian children aged 6-14 years: evaluation of Chaillet's international maturity standards. J. Forensic Leg. Med., 20(1): 40-5, 2013.

Gardner, E.; Simmons, M. J. \& Snutad, D. P. Principles of genetics. $8^{\text {th }}$ ed. New York, John Willey \& Sons, 1995.

Hegde, S.; Patodia, A. \& Dixit, U. A comparison of the validity of the Demirjian, Willems, Nolla and Häävikko methods in determination of chronological age of 5-15 year-old Indian children. J. Forensic Leg. Med., 50:49-57, 2017.

Lee, S. E.; Lee, S. H.; Lee, J. Y.; Park, H. K. \& Kin, Y. K. Age estimation of Korean children based on dental maturity. Forensic Sci. Int., 178(2-3):125-31, 2008.

Liversidge, H. L.; Smith, B. H. \& Maber, M. Bias and accuracy of age estimation using developing teeth in 946 children. Am. J. Phys. Anthropol., 143(4):545-54, 2010.

Martínez Gutiérrez, V. M. \& Ortega-Pertuz, A. I. Comparison of Nolla, Demirjian and Moorrees methods for dental age calculation for forensic purposes. Rev. Odontol. Mex., 21(3):e151-9, 2017.

Medina, A. C. \& Blanco, L. Accuracy of dental age estimation in Venezuelan children: comparison of Demirjian and Willems methods. Acta Odontol. Latinoam., 27(1):34-41, 2014.

Mohammed, R. B.; Krishnamraju, P. V.; Prasanth, P. S.; Sanghvi, P.; Lata Reddy, M. A. \& Jyotsna, S. Dental age estimation using Willems method: A digital orthopantomographic study. Contemp. Clin. Dent., 5(3):371-6, 2014. 
Nik-Hussein, N. N.; Kee, K. M. \& Gan, P. Validity of Demirjian and Willems methods for dental age estimation for Malaysian children aged 5-15 years old. Forensic Sci. Int., 204(1-3):208.e1-6, 2011.

Nur, B.; Kusgoz, A.; Bayram, M.; Celikoglu, M.; Nur, M.; Kayipmaz, S. \& Yildrim, S. Validity of demirjian and nolla methods for dental age estimation for Northeastern Turkish children aged $5-16$ years old. Med. Oral Patol. Oral Cir. Bucal, 17(5):e871-7, 2012.

Onat Altan, H.; Altan, A.; Bilgiç, F.; Akıncı Sözer, Ö. \& Damlar, I. The applicability of Willems' method for age estimation in southern Turkish children: A preliminary study. J. Forensic Leg. Med., 38:24-7, 2016.

Ortega-Pertuz, A. I. \& Piña-D`Abreu, M. S. Comparación de los métodos de Willems I y Willems II en la estimación forense de la edad en niños venezolanos. Rev. Esp. Med. Legal, 46(1):4-11, 2020.

Ortega-Pertuz, A. I.; Espina-Fereira, Á. I. \& Fereira-Paz, J. L. paplicability of Demirjian and Chaillet's methods in estimating dental age in children from the State of Zulia, Venezuela. Rev. Fac. Odontol. Univ. Antioq., 30(1):43-54, 2018.

Rodríguez-Larralde, A.; Castro de Guerra, D.; González-Coira, M. \& Morales, J. Frecuencia génica y porcentaje de mezcla en diferentes áreas geográficas de Venezuela, de acuerdo a los grupos RH y ABO. Interciencia, 26(1):8-12, 2001.

Schmeling, A.; Geserick, G.; Reisinger, W. \& Olze, A. Age estimation. Forensic Sci. Int., 165(2-3):178-81, 2007.

Urzel, V. \& Bruzek, J. Dental age assessment in children: a comparison of four methods in a recent French population. J. Forensic Sci., 58(5):1341-7, 2013.

Willems, G.; Van Olmen, A.; Spiessens, B. \& Carels, C. Dental age estimation in Belgian children: Demirjian's technique revisited. J. Forensic Sci., 46(4):893-5, 2001.
Dirección para correspondencia:

Dra. Ana Isabel Ortega-Pertuz

Área de Odontología Forense

Instituto de Investigaciones

Facultad de Odontología

Universidad del Zulia

Av. 19 con calle 65

Maracaibo, estado Zulia

VENEZUELA

Email: anitaortegav@gmail.com

Recibido : 27-01-2020

Aceptado: 20-03-2020 